\title{
Deconfinement Transition in Dual QCD
}

\author{
M. Baker \\ Department of Physics, University of Washington, Seattle, Washington 98195 \\ J. S. Ball \\ Department of Physics, University of Utah, Salt Lake City, Utah 84112 \\ and \\ F. Zachariasen \\ California Institute of Technology, Pasadena, California 91125 \\ (Received 11 April 1988)
}

\begin{abstract}
The QCD Lagrangean expressed in terms of dual potentials exhibits, at zero temperature, spontaneous symmetry breaking giving rise to dual superconductivity and therefore to color confinement. At a finite temperature, the spontaneous symmetry breaking disappears and so does confinement. We estimate the deconfining transition temperature using a high-temperature expansion.

PACS numbers: 12.38.Aw, 11.15.Ex, 11.15.Kc, 12.38.Lg
\end{abstract}

Color confinement in QCD is a phenomenon analogous to dual superconductivity (that is, superconductivity with electricity and magnetism interchanged). One should therefore expect that as the temperature is increased a transition is reached beyond which confinement goes away. This deconfinement transition has so far been studied mostly in the context of lattice gauge theory, ${ }^{1}$ though some analytic work based on mean-field theory has also been carried out. ${ }^{2}$

We have recently reformulated QCD using dual vector potentials $C_{\mu}^{a}$, variables which are better suited to the long-range confining regime than are the usual potentials $A_{\mu}^{a}{ }^{3}$ The long-range limit of the QCD Lagrangean, $\mathcal{L}(C)$, expressed in terms of the $C_{\mu}^{a}$, turns out to describe a non-Abelian dual superconductor. Confinement results from spontaneous symmetry breaking associated with a magnetic condensate. Color electric flux can exist only in the form of confined quantized $Z_{N}$ flux tubes.

The QCD Lagrangean, expressed in terms of dual potentials, provides an explicit framework in which to study quantitatively phenomena associated with the confining regime. In particular, one can study the deconfining transition, and that is the primary purpose of this paper.

The physics of deconfinement can be understood in the context of pure (dual) glue alone, and we shall study the transition only in the absence of quarks, or, in other words, for infinite quark mass. The reason for this is that it is awkward to couple dynamical quarks to dual gluons, just as it is awkward to couple magnetic monopoles to photons in ordinary quantum electrodynamics. But while the inclusion of quarks is awkward, it is not impossible, and we hope to extend our discussion to finite quark masses in a later publication.

We will calculate the deconfinement transition temperature in the high-temperature limit, since only in this limit can the calculation be carried out analytically. The transition temperature we obtain, however, does not turn out to be high enough so that this approximation is very good, and our numerical result must therefore be regarded only as an estimate.

The Lagrangean $\mathcal{L}(C)$ constructed in I is supposed to describe QCD in the long-range confining regime. It is characterized by a mass scale $M$ (in principle related to $\Lambda_{\mathrm{QCD}}$ ) which we have estimated to be about $450 \mathrm{MeV}$. We expect that, at zero temperature and for energies below $M, \mathcal{L}(C)$ describes $\mathrm{QCD}$ correctly.

Confinement in dual QCD is, as mentioned above, characterized by a nonperturbative vacuum containing a magnetic condensate proportional to $\tilde{F}_{0}^{2} \equiv\left\langle\tilde{F}_{\mu \nu}^{a} \tilde{F}_{\mu \nu}^{a}\right\rangle$. In the confined regime, dual gluons (and indeed all dynamical degrees of freedom) have a nonzero mass. Therefore the dual gluon field exhibits only short-range correlations, the dual Wilson loop obeys a perimeter law, and so the ordinary Wilson loop obeys an area law. ${ }^{4}$

When the deconfinement temperature $T_{d}$ is reached, $\tilde{F}_{0}^{2}$ vanishes. There is no longer any spontaneous symmetry breaking and the vacuum is perturbative. Dual gluons now develop zero-mass singularities, so that color electric fields can spread out and are not confined to tubes of quantized flux. Therefore the perimeter law for the dual Wilson loop fails, and so does the area law for the ordinary Wilson loop.

The dual QCD Lagrangean, without quarks, is

$$
\mathcal{L}(C)=2 \operatorname{Tr}\left\{\frac{1}{2} M G_{\mu \nu} \tilde{F}_{\mu \nu}+\frac{1}{4} \tilde{F}_{\mu \nu} D^{2}(C) \tilde{F}_{\mu \nu}-\frac{1}{4} G_{\mu \nu} G_{\mu \nu}-W(\tilde{F})\right\} .
$$

Here $G_{\mu \nu}$ is the dual field tensor,

$$
G_{\mu \nu}=\partial_{\mu} C_{v}-\partial_{\nu} C_{\mu}-i g\left[C_{\mu}, C_{v}\right],
$$

(C) 1988 The American Physical Society 
and $\mathscr{D}_{\mu}(C)$ is the dual covariant derivative

$$
\mathcal{D}_{\mu}(C)=\partial_{\mu}-i g\left[C_{\mu},\right],
$$

where $C_{\mu}$ is the dual potential and $g$ is the magnetic coupling constant, related to the usual Yang-Mills coupling constant $e$ via $e g=2 \pi$. The space-space components of $G_{\mu \nu}$ are the color electric displacement vector D,

$$
G_{i j}=\epsilon_{l j k} D_{k}
$$

and the space-time components are the magnetic $\mathbf{H}$ field,

$$
G_{0 i}=H_{i} \text {. }
$$

The tensor $\tilde{F}_{\mu \nu}$ gives the electric $E$ and magnetic $B$ fields (strictly, the polarization fields):

$$
\begin{aligned}
& \tilde{F}_{0 i}=-B_{i} / M, \\
& \tilde{F}_{i j}=-\epsilon_{i j k} E_{k} / M .
\end{aligned}
$$

In all of the above equations we use the notation $C_{\mu}=\sum_{a} C_{\mu}^{a} T_{a}$, where the generators $T_{a}$ of the color gauge group satisfy

$$
\left[T_{a}, T_{b}\right]=i f_{a b c} T_{c}
$$

and are normalized to

$$
2 \operatorname{Tr}\left(T_{a} T_{b}\right)=\delta_{a b} .
$$

The function $W(\tilde{F})$ contains the counterterms necessary to make $\mathcal{L}$ renormalizable. There are counterierms quadratic and quartic in $\tilde{F}$. We have, for $\operatorname{SU}(N)$,

$$
W(\tilde{F})=-\left(\mu^{2} N / 4\right) \tilde{F}_{\alpha \beta j}^{i} \tilde{F}_{\alpha \beta i}^{j}+(N \lambda / 4 !)\left\{\tilde{F}_{\alpha \beta}{ }_{j}^{i} \tilde{F}_{\alpha \beta} \dot{k} \tilde{F}_{\gamma \delta} k^{k} \tilde{F}_{\gamma \delta i}^{l}+(1 / N)\left(\tilde{F}_{\alpha \beta}^{i} \tilde{F}_{\alpha \beta i}^{j}\right)^{2}+(2 / N)\left(\tilde{F}_{\alpha \beta}^{i} \tilde{F}_{a \beta}^{k}\right)\left(\tilde{F}_{\gamma \delta i}^{j} \tilde{F}_{\gamma \delta} k\right)\right\},
$$

where $F_{a \beta} i / \sqrt{2}$ is the $i j$ matrix element of the color matrix $F_{\alpha \beta}$ [the factor $1 / \sqrt{2}$ is due to the normalization choice Eq. (9)].

$W(\tilde{F})$ plays the role of the Higgs potential in dual QCD. Clearly $\lambda$ must be positive for stability. Then spontaneous symmetry breaking always occurs and $W(\tilde{F})$ has a minimum at a nonzero value of $\tilde{F}_{0}^{2}$ $\equiv F_{0} \underset{a \beta}{a} F_{0 a \beta}^{a}$, which determines the gluon condensate $G_{2}=-(M / g)^{2} \tilde{F}_{0}^{2}$. The value of $W$ at the minimum is the vacuum energy density $\epsilon_{\mathrm{vac}}$. Minimizing Eq. (10) yields

$$
\epsilon_{\mathrm{vac}}=\left(N g^{2} / 8\right)(\mu / M)^{2} G_{2} .
$$

Comparing Eq. (11) with the relation between $\epsilon_{\mathrm{vac}}$ and $G_{2}$ given by the QCD trace anomaly, we find

$$
-\mu^{2}=\frac{11}{12}(M / g)^{2} .
$$

Thus asymptotic freedom (used to evaluate the QCD trace anomaly) forces $\mu^{2}$ to be negative. Using Eq. (12) and the value of $\tilde{F}_{0}^{2}$ obtained from minimizing Eq. (10), we can calculate $G_{2}$ :

$$
G_{2}=\frac{11}{8 \lambda}\left(\frac{M}{g}\right)^{4} \frac{N^{2}-1}{N} .
$$

The vacuum is therefore magnetic $\left(G_{2}>0\right)$ as is to be expected for a dual superconductor, and $\epsilon_{\mathrm{vac}}$ is negative so that the nonperturbative vacuum has a lower energy than the perturbative vacuum, in which $\tilde{F}_{0}^{2}=0$.

When the temperature is finite there are additional contributions to the vacuum energy density. Some of these are proportional to $\tilde{F}_{0}^{2}$, so that for finite $T$, the quadratic part of $\left.W(\tilde{F})\right|_{\tilde{F}^{2}=\tilde{F}_{0}^{2}}$ has the form

$$
-\left(\mu^{2} N / 4\right) \tilde{F}_{0}^{2}+f(T) \tilde{F}_{0}^{2} \equiv-(N / 4) \mu^{2}(T) \tilde{F}_{0}^{2},
$$

where $f(T)$ vanishes at $T=0$ and is positive for finite $T$. The critical temperature for the deconfining transition is $T_{c}$, where $f\left(T_{c}\right)=\mu^{2} N / 4$; at this temperature the sign of the quadratic term in $W(\tilde{F})$ changes and the minimum of $W$ occurs at $\tilde{F}_{0}^{2}=0$. Above $T_{c}$ the perturbative vacuum obtains, spontaneous symmetry breaking disappears, and confinement is lost.

At high temperature and for weak coupling the dominant contributions to $f(T)$ come only from one-loop graphs. As can be seen from the dual Lagrangean (1), there are only two of these, shown in Fig. 1. The vertices can be read off from (1), and the contribution of these graphs to the internal energy density is

$$
\frac{\Omega}{V}=\frac{1}{2 \beta} \sum_{n=-\infty}^{\infty} \int \frac{d^{3} k}{(2 \pi)^{3}}\left\{-\frac{g^{2} N}{2} \tilde{F}_{0}^{2} \Delta_{\mu \mu}^{a a}(k)+X_{a \beta, \gamma \delta}^{a a} \Delta_{a \beta, \gamma \delta}^{a a}(k)\right\} .
$$

The two terms here correspond to Figs. 1 (a) and 1(b), respectively. $\Delta_{\mu \nu}^{a b}(k)$ is the dual-gluon propagator, and $\Delta_{\alpha \beta, \gamma \delta}^{a b}(k)$ is the propagator of the $\tilde{F}$ field. These are the propagators in the absence of the potential ${ }^{5} W(\tilde{F})$; they are given explicitly in Eqs. (3.15) and (3.17) of I. The quantity $X_{a \beta, \gamma \delta}^{a b}$ gives the coupling to the $F$ field, and is defined by

$$
X_{a \beta, \gamma \delta}^{a b}=-\left.\frac{1}{2} \frac{\partial^{2} W_{4}(\tilde{F})}{\partial \tilde{F}_{a \beta}^{a} \partial \tilde{F}_{\gamma \delta}^{b}}\right|_{\tilde{F}=\tilde{F}_{0}},
$$

where $W_{4}$ is the quartic (in $\left.\tilde{F}\right)$ part of $W$. Finally, the four-vector $k$ appearing in the integral in (14) is $k=\left(z_{n}, \mathbf{k}\right.$ ), 


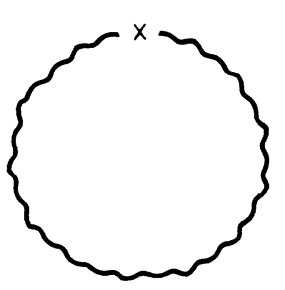

(a)

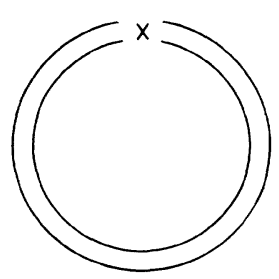

(b)

FIG. 1. Graphs showing the contribution of the (a) $\tilde{F}_{0}^{2} C^{2}$ term and (b) $\tilde{F}^{2} \partial^{2} W /\left.\partial \tilde{F}^{2}\right|_{\tilde{F}^{2}=\tilde{F}_{0}^{2}}$ term in $\mathcal{L}(C)$ to the vacuum energy density at finite temperature. The wavy line is a dual gluon; the double line is the $\tilde{F}$ field.

where $z_{n}=(2 \pi i / \beta) n$.

The sum and integral in (14) are readily evaluated in the high-temperature limit. In this limit, we obtain

$$
\Omega / V=\left[-\left(g^{2} N / 16\right) \tilde{F}_{0}^{2}+\frac{1}{6} X\right] T^{2},
$$

where

$$
X \equiv X_{\alpha \beta, \alpha \beta}^{a a}
$$

$X$ may be evaluated with use of its definition and (10); we obtain

$$
X=-\frac{1}{3} \lambda\left(16+15 N^{2}\right) \tilde{F}_{0}^{2} .
$$

Finally, putting all of this together we arrive at the following expression for the temperature-dependent mass $\mu^{2}(T)$ in the high-temperature approximation:

$$
-\frac{N}{4} \mu^{2}(T)=-\frac{N}{4} \mu^{2}-T^{2}\left(\frac{g^{2} N}{16}+\frac{\lambda}{18}\left(16+15 N^{2}\right)\right) .
$$

$$
\begin{aligned}
& \Delta_{\mu \nu}^{a b}=\delta^{a b} \frac{q^{2}-M^{* 2}}{q^{2}\left(q^{2}-M^{2}-M^{* 2}\right)}\left(\delta_{\mu \nu}-\frac{q_{\mu} q_{v}}{q^{2}}\right), \\
& \Delta_{\mu, \gamma \delta}^{a b}=\delta^{a b} \frac{i M}{q^{2}\left(q^{2}-M^{2}-M^{* 2}\right)}\left(q_{\gamma} \delta_{\mu \delta}-q_{\delta} \delta_{\mu \gamma}\right), \\
& \Delta_{a \beta, \gamma \delta}^{a b}=\left\{\frac{1}{q^{2}-M^{* 2}}\left(\delta_{\alpha \gamma} \delta_{\beta \delta}-\delta_{\alpha \delta} \delta_{\beta \gamma}\right)+\frac{M^{2}\left(q_{\alpha} q_{\gamma} \delta_{\beta \delta}-q_{\alpha} q_{\delta} \delta_{\beta \gamma}-q_{\beta} q_{\gamma} \delta_{\alpha \delta}+q_{\beta} q_{\delta} \delta_{\alpha \gamma}\right)}{q^{2}\left(q^{2}-M^{* 2}\right)\left(q^{2}-m^{2}-M^{* 2}\right)}\right\},
\end{aligned}
$$

where $M^{* 2}=N \mu^{2}(T)$. At $T=0, \mu^{2}(T)<0$, but above $T_{d}, \mu^{2}(T)>0$ so that in the perturbative vacuum, where all of this applies, $M^{*}$ represents a normal real mass, not a ghost. Note the presence of zero-mass singularities in the dual-gluon propagator.

Eliminating the $\tilde{F}$ field using the field equations, we can write the quadratic part of the dual Lagrangean as

$$
\mathcal{L}(C)=-\frac{1}{4}\left(\partial_{\mu} C_{\nu}^{a}-\partial_{\nu} C_{\mu}^{a}\right) \mu\left(\partial_{\mu} C_{\nu}^{a}-\partial_{\nu} C_{\mu}^{a}\right),
$$

and the permeability $\mu$ can be identified. In momentum
The deconfinement temperature $T_{c}$ is the point at which $\mu^{2}(T)$ vanishes; thus

$$
T_{c}^{2}=\frac{-N \mu^{2} / 4}{g^{2} N / 16+\lambda\left(16+15 N^{2}\right) / 18} .
$$

The parameters appearing in (19) are all approximately known from the analysis given in I. The uncertainty in their values arises from the fact that some of the analysis is done for $\mathrm{SU}(2)$ rather than $\mathrm{SU}(3)$ as the color gauge group. With use of the parameters given in I, Eq. (19) gives a deconfinement temperature of 80 $\mathrm{MeV}$. This value is too low for the high-temperature approximation used in obtaining it to be valid, since the typical mass scale in the problem, as indicated in I, is about $175 \mathrm{MeV}$. It must therefore be regarded as an estimate only. In fact, lattice calculations give a number more like $150 \mathrm{MeV}$ (or even $250 \mathrm{MeV}$ ), twice the value that we obtain. ${ }^{1}$

The deconfinement transition calculated here is second order. However, the simple high-temperature approximation fails near the critical point, and higher-order effects need to be included. ${ }^{6}$ We have not done this, and so the question of whether the transition is first order or second order is not settled. (We note that lattice calculations suggest that the transition is first order. ${ }^{1}$ )

As we mentioned in the introduction, we expect $\mathcal{L}(C)$ to be valid up to energies, or temperatures, of order $M$. Since $M$ is larger than $T_{c}$, it should be possible to use $\mathcal{L}(C)$ to describe the deconfined plasma in the temperature range between $T_{c}$ and $M$. In this regime, the perturbative vacuum obtains. The propagators for the dual gluon and $\tilde{F}$ fields can be derived from the quadratic part of $\mathcal{L}(C)$, Eq. (1), with $\tilde{F}_{0}^{2}=0$, and turn out to be the following (in Landau gauge): space, we find

$$
\mu=\epsilon^{-1}=\left(q^{2}-M^{2}-M^{* 2}\right) /\left(q^{2}-M^{* 2}\right) .
$$

When $q^{2}>M^{2}$, the dielectric constant approaches unity. Therefore for $T \gg M$, conventional nondual perturbative asymptotically free QCD describes the plasma. There are thus, in effect, three regimes. From $T=0$ to $T=T_{c}$, there is the confined regime, described by $\mathcal{L}(C)$ with $\tilde{F}_{0}^{2} \neq 0$. From $T_{c}$ to a temperature comparable to $M$, 
$\mathcal{L}(C)$ can still be used, but now with the perturbative vacuum having $\tilde{F}_{0}^{2}=0$. This is a sort of an intermediate regime, with no confinement because the $A$-language Wilson loop does not obey an area law, but not the realm of perturbative QCD either. Above $M, \mathcal{L}(C)$ reduces to the usual Yang-Mills Lagrangean in the $A$ language; this is the regime of asymptotic freedom.

The dielectric constant summarizes the effect of the interactions of dual gluons among themselves in the perturbative vacuum. When quarks are included, one can then think of the quark-gluon plasma as essentially the same as an ordinary electrical plasma, containing electrons and positrons interacting electromagnetically in a medium with the dielectric constant (24). Such a medium has some unusual properties, and the plasma, consequently, has features differing from those of a conventional electron plasma. For example, the high-temperature dispersion relation for long-wavelength plasma oscillations, ${ }^{7} \omega^{2}=e^{2} T^{2} / 6$, is modified to

$$
\epsilon(\omega) \omega^{2}=N_{f} e^{2} T^{2} / 6
$$

where $N_{f}$ is the number of flavors. The using of (24) for $\epsilon$ gives rise to plasma instabilities (complex $\omega^{2}$ ) for $N_{f} e^{2} T^{2} / 12$ in the range $M^{2}+\frac{1}{2} M^{* 2} \pm M\left(M^{2}\right.$ $\left.+M^{* 2}\right)^{1 / 2}$. Recall that $M^{*}$ vanishes at the deconfinement point. For temperatures not too far above this, we may expect $M^{* 2} \ll M^{2}$. The range of instabilities is therefore $N_{f} e^{2} T^{2} / 12$ roughly between $M^{* 4} / 8 M^{2}$ and $2 M^{2}$, or $T_{c}<T<\left(6 / \pi \alpha_{s} N_{f}\right)^{1 / 2} M$. Numerically, with use of the parameters given in $I$, the upper limit here is about $900 \mathrm{MeV}$. Therefore the instabilities would ap- pear to exist over the entire range of temperatures in which the unconfined perturbative form of $\mathcal{L}(C)$ might be valid. Thus the usual quark-gluon plasma may really only exist in the asymptotically free regime, for temperatures above $M$.

Further, more detailed speculations based on the use of the dual language to describe the deconfined phase will be reported elsewhere.

We are indebted to R. Norton for advice on the use of finite-temperature field theory, and to the Aspen Center for Physics, where this work was begun, for its hospitality. The work of M.B. was supported in part by U.S. Department of Energy Contract No. DEAC06-81ER40008 , that of J.S.B. was supported in part by National Science Foundation Grant No. PHY-8706501, and that of F.Z. was supported in part by U.S. Department of Energy Contract No. DEAC-03-81-ER40050.

\footnotetext{
${ }^{1}$ R. Gupta et al., Los Alamos Report No. LA-UR-87-3068, 1987 (to be published); J. Kogut et al, Phys. Rev. Lett. 55, 1475 (1985), and 50, 393 (1983); J. Cleymans et al., Phys. Rep. 130, 217 (1986).

${ }^{2}$ J. J. Kapusta, Nucl. Phys. B190, 425 (1981); B. Müller and J. Rafelski, Phys. Lett. 101B, 111 (1981).

${ }^{3}$ M. Baker, J. Ball, and F. Zachariasen, Phys. Rev. D 37, 1036,3785 (E) (1988); hereafter referred to as I.

${ }^{4}$ G. 't Hooft, Nucl. Phys. B153, 141 (1979).

${ }^{5}$ L. Dolan and R. Jackiw, Phys. Rev. D 9, 3320 (1974).

${ }^{6}$ S. Weinberg, Phys. Rev. D 9, 3357 (1974).

${ }^{7}$ D. Gross et al., Rev. Mod. Phys. 53, 1 (1977).
} 